\title{
Multi-peptide CMV-Modified Vaccinia Ankara Vaccine
}

National Cancer Institute

\section{Source}

National Cancer Institute. Multi-peptide CMV-Modified Vaccinia Ankara Vaccine. NCI

Thesaurus. Code C111036.

A vaccine consisting of an inactivated, Modified Vaccinia Ankara (MVA) viral vector encoding three herpes virus cytomeg alovirus (CMV) tumor-associated antigens (TAAs), including UL83 (pp65), UL123 (IE1) and UL122 (IE2), with potential immunostimulating activity. The viral peptides expressed after administration of the multi-peptide CMV-MVA vaccine, may stimulate the immune system to mount both cytotoxic T-lymphocyte $(C T L)$ and helper T-cell responses against CMV-infected cells. This results in cell lysis and prevents viral replication and the development of CMV disease. This vaccine also provides active immunization and protective immunity against CMV infection in CMV-negative patients. CMV infection can cause serious complications in patients receiving either allogeneic hematopoietic cell transplants (HCT) or solid org an transplants. 